\title{
MANIPULATION:
}

\section{INDIVIDUAL RESPONSIBILITY}

As physiotherapists we have a particular responsibility to the community. Primarily this responsibility is to our patients and to the doctors who refer them. When we consider our responsibility in relation to manipulation, we need to think of two particular aspects. Firstly it is our responsibility to see that such treatment is available to the community as a part of routine ethical medical care. By providing this treatment we render it unnecessary for people to resort to treatment outside the ethical medical umbrella. To provide this treatment we need adequate under-graduate training with opportunities for further training at the post-graduate level. Secondly, we have very definite responsibilities when we treat patients. These include competence in examination, treatment techniques and assessment. During treatment we must be fully aware of all changes which take place so as to be able to act responsibly for the doctor concerned.

It is not the intention of this paper to discuss postgraduate and under-graduate training, given as the first responsibility, because it is assumed we all recognise that manipulation is a part of physiotherapy and that we are doing our best to fulfil the demands which this statement implies. Also, the subject has been discussed at length elsewhere. ${ }^{i}$ The individual physiotherapist's responsibility is the concern of this paper. Some physiotherapists may feel they are unable to further the acceptance and general use of manipulative treatment either because they are not in a position to do so or because they feel they cannot muster the co-operation of the physiotherapy profession as a whole. Nevertheless there are things which each person can undertake. We have responsibilities in regard to manipulation about which each of us can do something and none of us, with a clear conscience, can pass them by. These individual responsibilities relate to the way we examine, treat and assess our patients, and the co-operation we develop with the doctors for whom we work. These factors can be discusserl under the following headings:

\section{OBJECTIVITY AND METHOD}

(i) Recording

(ii) Examination

(iii) Assessment

\section{RESPONSIBILITY}

(i) Physiotherapist to doctor

(ii) Neurological examination.

\section{OBJECTIVITY AND METHOD}

If physiotherapists were always objective and methodical in the application and interchange of techniques in the treatment of every patient there would never be need to justify the place of physiotherapy in community medical care. It might also be said that if we were always objective and methodical, much of the wasteful time spent on treatment would be eliminated. Our methodical approach to treatment should follow the same routine whether we are using one particular method of heat treatment, one particular type of exercise, or one technique of manipulation. The routine should be as follows.

1. Before commencing each treatment a clear correct assessment of the patient's symptoms and signs should be made against which progress can be assessed.

2. One technique of treatment should then be applied, the details of which should be recorded together with any effect it has had during application.

1. Hammond, M. J. and Maitland, G.D. (1969). Teaching Manipulation to Post-graduate and Under-graduate Students. N.Z. J. Physiother. $3(16), 4$.

\section{G. D. MAITLAND}

A.U.A., M.A.P.A., F.C.S.P.

3. Following the application of this technique a reassessment of the patient's symptoms and signs should be made. These new findings are recorded opposite the recording of the technique to indicate the changes which have taken place as a result of the technique.

This principle of breaking treatment up into segments for the purpose of proving the value of each segment should be the routine for each and every treatment, no matter whether it is applied to the patient who is paraplegic, the patient who has metarsalgia or the patient who has pain in his back. This is our first responsibility.

Two reasons are commonly given for not. using this methodical routine, "it takes too long" and "some conditions are too slow in their response to treatment to assess in this way". The latter may be a reason for not doing it in detail following each application of each technique at each treatment. However the principle still holds, though it takes longer to "prove the value" of the technique. As to the matter of insufficient time, this statement is incorrect. If abbreviations are used and a routine of recording adopted, the method is not time consuming. Even if it did take time, omission of this routine would not be justified.

As physiotherapists we learn many manual techniques but competency in techniques is not enough, our responsibility goes further. The techniques should be used in the routine described above to prove the value of each technique in treatment. Together with competency with techniques and the methodical application of these techniques there are three further components of responsible treatment, recording, examination and assessment.

\section{Recording}

In this paper emphasis is put on "Recording" because, by recording findings properly, the position is more readily clarified. It is obvious that the examination findings determined during the patient's first visit will be recorded in detail. Treatment involving the methodical use of one technique at a time referred to above must also be recorded and the following routine is suggested.

1. Before each treatment a brief record is made of:

(i) the effect of previous treatment or treatments on the patient's symptoms;

(ii) the effect of previous treatment or treatments on the patient's joint signs.

2. During treatment the technique used is recorded with brief notes made regarding:

(i) which joint is treated;

(ii) which technique is used, together with some indication of how strongly it is performed;

(iii) any symptoms which the patient may feel while the technique is being applied.

3. After the technique has been used, the patient's symptoms and signs should be reassessed so that any changes found can be recorded opposite the record of treatment, thus indicating the effect of the treatment.

Table 1 gives a summary of these details and shows how briefly it can be done. The recording is simple, concise and most effective, and once it becomes habit it is very quick. 
Table 1

C/O-Subjective assessment. Interpret what the patient says about his symptoms. Check on main points. With patients whose progress can be expected to be slow, make the comparison over a week, rather than 24 hours.

$\mathrm{O} / \mathrm{E}$-Objective assessment. Check important joint signs.

Plan-State why you choose a certain technique/exs/heat.

$\mathrm{T} / \mathrm{T}$-State the techniques used, the strength at which it was done,

the joint to which it vas done, the number of times it was done, and the effect it had while being performed

Plan-State reasons for any change which you consider may be required and note any reminders for the next treatment.

\section{Example}

C/O-"Moving more freely, but more back pain."

$\mathrm{O} / \mathrm{E}-\mathrm{F}=6^{\prime \prime}$ less pain.

LF (B) $\sqrt{ }$ slight pain with o/pressure

Plan-Repeat last $t / t$ because rate of progress adequate.

$\mathrm{T} / \mathrm{T}--2 \times \rightarrow$ III L1 -3

no pain

$\mathrm{C} / \mathrm{O}$ "Movement easier"

$\mathrm{O} / \mathrm{E} \mathrm{F}=3^{\prime \prime}$ sl. back p.

U.S. $1.5 \mathrm{w} / \mathrm{cm}^{2} \times 7^{\prime} \mid$ I.S.Q.

If progress not maintained, may be worth a trial of traction.

\section{Examination}

Treatment can only be applied accurately if examination is accurate. The detail required for the examination of joint movements is greater than most physiotherapists appreciate. The referring doctor, with his responsibility for diagnosing the condition, does not require the finer details regarding movement which are necessary for the physiotherapist if she is to use manipulation. The detail of joint movement we require includes the extent and behaviour of pain, of stiffness and of muscle spasm with each direction of movement.

The behaviour of pain with movement is very important, as is borne out in the following examples of equally restricted lumbar flexion, each with a different pain pattern. The differences are important because they guide the treatment and because, if they are not appreciated, the patient may be made worse by treatment without the physiotherapist realising. In all of the examples which follow, the patient has an ache in his lower back which extends down his leg into his calf. On forward flexion, he first feels a change in his pain when his finger-tips reach his knees. With further movement he can reach halfway down his shin with the following differences in the behaviour of his pain.

(i) There is no alteration to the pain, "half-shin" being the normal limit of his range.

(ii) His back pain increases in intensity until the increase in this pain prevents him from flexing further than half-way down his shin. His thigh and calf symptoms are not affected by the flexion.

(iii) As movement increases the pain spreads into his buttock but it is stiffness which prevents him from flexing further than half-way down his shin.

(iv) As movement increases so the pain spreads down his leg to his calf.

(v) As movement increases the pain in his back disappears but his calf becomes increasingly painful as the movement reaches the limit of his range.

When pain behaves as indicated in (v) the patient must be treated with much more care than when it behaves in a manner similar to (i). The first indicates a nerve root pain which may be harmed by too zealous treatment, while the other is less likely to be worsened by treatment. If we are treating a patient such as in (i) and do not take note of the behaviour of pain throughout movement then his pain may change to being that of (v) without our appreciating it.
Behaviour of pain is but one aspect of examination requiring care with detail. Movement is another. We should observe how the individual segments of the vertebral column move during examination, particularly when the movement is painful. Just as it is normal for us, when examining elevation of the shoulder, to watch the scapulo-thoracic rhythm of movement, so we should also watch intervertebral movement when examining movements of the vertebral column. Appreciation of gross range alone is inadequate.

Any joint which is not causing symptoms should be able to accept a certain amount of stretch at the limit of its ranges without pain, and we must know the strength of the stretch which can be applied without pain to the different movements of each joint. Therefore, when examining joint movements which appear normal, we should apply an adequate stietch before classing it as normal. It is surprisingly commen for joint signs of a minimal nature to pass unnoticed because physiotherapists do not appreciate how strong a stretch to apply. This is a common cause of error in examination among physiotherapists.

It is important that examination should include all joints and muscles which might be causing part or all of a patient's symptoms. If the present discussion is confined to the source of pain, omitting reference to all other aspects such as posture, muscle weakness, etc., the examination should include inspection of the following:

1. The joints which lie under the area of pain.

2. The joints which do not lie under the area of pain but can refer pain into the area.

3. The muscles which lie under the area of pain.

An important part of examination for conditions of the vertebral column is based on movement of the intervertebral joint determined by pressure against palpable parts of the vertebra. By these pressures it is possible to determine loss of range and the behaviour of pain, muscle spasm and stiffness during movement in all directions. These movements include both physiological movements and accessory movements. Physiotherapists hoping to use gentle mobilising techniques which can be localised to one joint, must learn how to use these techniques both for examination purposes and for treatment. Once competency has been achieved with these techniques, assessment becomes far more accurate for conditions where pain arises from the spine.

When we think of movements, we should not forget movements of the pain sensitive structures within the vertebral canal. There are few movements which will move these structures without at the same time moving the joints of the spine. However movements such as flexing the head and neck of the patient lying supine tests movement of the canal structures in the lumbar and thoracic spines, prone lying knee flexion tests movement of the nerve roots and their sleeves in the upper lumbar area and straight leg raising tests movement of the lumbo-sacral nerye roots and their sleeves. Combinations of straight leg raising, neck flexion, dorsi-flexion of the ankle and trunk flexion can be used to increase tension in the structures.

\section{Assessment}

Assessment is the hinge around which constructive treatment revolves. Although treatment can be administered without examination and assessment it will not be constructive and cannot be informative. At the initial examination it is not possible to predict which techniques will relieve a patient of his pain and guess work in the choice of techniques can only succeed by chance. To rely solely on the directions of the referring doctor does not allow for unpredicted changes. If, during treatment, the patient is not improving, changes in technique should be made, but it is not possible to know when to make such changes if repeated assessment is not a normal part of treatment. Nor is it possible to know accurately without assessment when treatment should be discontinued. Therefore "assessment" is the hinge around which constructive treatment revolves.

The first assessment is that made preceding each day's treatment. It should include an appraisal of the patient's 
opinion of the effect of treatment on his symptoms, and an assessment of changes of his joint signs resulting from previous treatment. The second assessment is that made during each treatment session. It is made following the use of individual techniques to prove the value of that particular technique. It is difficult to teach the extent of improvement in symptoms and signs which indicates that a particular technique should be continued or when changes should be made. Much of the judgement influencing changes in techniques comes only with experience but experience will only provide the basis for this knowledge if the habit of assessment is established.

We are not always able to improve a patient's symptoms and signs. However it is extremely valuable if the physiotherapist is able to tell the doctor confidently that continued physiotherapy will not produce any further improvement in the patient's symptoms or signs. The doctor is then in a position to make clearer judgements regarding the further management of his patient. When physiotherapists are able to do this the profession will be fulfilling its role more successfully.

\section{RESPONSIBILITY}

\section{Physiotherapist to doctor}

If the patient is to receive the best possible care, it is essential that we work in very close co-operation with the medical profession. Regarding manipulation this is particularly relevant with specialists in orthopaedics, neurosurgery and physical medicine. It is essential that the medical practitioner should be able to refer his patients confidently for physiotherapy knowing that manipulative treatment will be gentle and that very careful assessment of progress will be

\section{THE LUMBO-SACRAL SPINE}

\section{An Approach to Treatment}

"The spine is a bunch of bones. Your head sits on one end and you sit on the other." Art Linkletter in "Kids Sure Rite Funny!"

It is customary to start an article with a review of the literature on the subject, a description of techniques used, followed by illustrative case histories and discussion. ${ }^{1}$ As this is such a vast subject it is proposed to start with the description of a patient and her response to treatment and then follow with a discussion of some of the points which arise.

\section{THE PATIENT}

On 29th February, 1972, a married Coloured female aged 26 years was referred to the manipulation unit for mobilisation of her lumbar spine. The diagnosis was "lumbosacral breakdown". She was examined by a physiotherapist on 1st March, 1972.

\section{Symptoms}

She complained of pain across the lumbo-sacral junction spreading at times into both legs (see Fig. 1 "Body Chart"). The pain spread farther down the left leg and it was more painful than the right. She also said that she had intermittent paraesthesiae in the lateral aspects of both thighs. The backache was a constant deep ache, worse than the leg pain. There were no other symptoms. Her backache was aggravated by sustained flexion, sitting for two or three hours (e.g. at a cinema), getting up from sitting, turning over at night, long sitting (e.g. in a bath) and upright kneeling. On waking in the moring her backache had eased, but was worse again a few minutes after getting up. Coughing hurt her back. ${ }^{2}$

The leg pain and pins and needles came on after sitting for two or three hours, was worse on the left side and caused her to limp for a few steps after standing up because of pain made throughout treatment. By undertaking treatment of his patients we are accepting a very real responsibility. If we accept this responsibility properly the medical profession will be able to make use of our faculties of observation and assessment to great advantage.

\section{Neurological Examination}

One aspect of our responsibility warrants particular emphasis. It applies to our ability to carry out a reliable neurological examination to determine alteration in nerve conduction due to compression of the nerve root. It is vital that we should be able to do this competently so that we can watch progress. We should not undertake treatment of patients by manipulation without competence in neurological examination.

\section{CONCLUSION}

It is generally accepted that manipulation is part of physiotherapy, and thus we accept certain responsibilities. These include responsibilities as a profession and responsibilities as individuals.

As a normal routine, patients must be examined with sufficient accuracy and detail to enable the effect of treatment to be assessed and the value of individual techniques proven. Obviously it is necessary to achieve competency in the use of many techniques to manipulate. We also need to cultivate the habit of assessing the value of each technique used in treatment. This habit will do more good for physiotherapy than any other single facet of our work.

Finally, we have a very important responsibility to work closely with the doctors who refer patients for treatment, and it is by this means that manipulation, as a part of ethical medical care, will proceed in the right manner. and a "lame" feeling in her legs. After a few minutes the leg pain disappeared and the backache eased a little.

Prone lying eased her back pain and she preferred to sleep prone on a bed with a board and inner-spring mattress. Slight tilting of her pelvis towards flattening of the lumbar spine in standing also eased her backache a little. There was no latent pain and the joint was not thought to be irritable. ${ }^{3}$ She had no symptoms of cauda equina compression ${ }^{4,5}$ and her general health was good with no recent weight loss. "She had had an infection of the cervix which had caused abdominal pain four years previously.

She was taking Brufen, Doloxene and Beserol which she said did not help her. X-rays taken on 22nd February, 1972, were reported normal. The fifth lumbar vertebra was set rather high relative to the iliac crests in the $A-P$ view. ${ }^{7,8}$ There was a slight tilt to the right at $14 / 5$ disc space, with a widening on the left. ${ }^{2}$ The lumbosacral facet joints were asymmetrical, the right being more saggittal and the left more frontal. $5,7,8,9$

\section{History}

She had fallen onto her buttocks in 1969 when she was seven months pregnant but had felt no pain then. In August, 1970, she bent forward and was fixed in flexion with severe pain in her back. 2,10 She managed to get to bed and on rising the next morning could straighten up but had back pain which radiated to both legs as far as the knees. She was referred to the physiotherapy department where she was examined by a doctor on 21 st September, 1970. The pain then was aggravated by bending and lifting and relieved by rest. Flexion was limited and sacro-iliac joint tests were positive on the left. Straight leg raising, neurological tests and X-Rays were normal. (See Fig. 2 "Examination Record".) 